\title{
Investigation of Coronal Leakage of Root Fillings After Smear-Layer Removal with EDTA or Nd:YAG Lasing Through Capillary-Flow Porometry
}

\author{
Rafaël Michiels, D.D.S., Tom Edgard Maria Vergauwen, D.D.S., Athina Mavridou, D.D.S., M.Sc., \\ Maarten Meire, D.D.S., M.Sc., Mieke De Bruyne, D.D.S., Ph.D., \\ and Roeland Jozef Gentil De Moor, D.D.S., M.Sc., Ph.D.
}

\begin{abstract}
Objective: This study investigates the effects of Nd:YAG laser irradiation combined with different irrigation protocols on the marginal seal of root fillings. Background data: Limited information exists regarding the effects of morphologic changes to root canal (RC) walls after Nd:YAG laser irradiation after smear-layer removal with EDTA on the sealing ability of root fillings. Methods: The 75 root-filled teeth $(5 \times 15$ teeth) were analyzed for through-and-through leakage by using capillary flow porometry (CFP). The RC cleaning procedure determined the assignment to a group: (1) irrigation with $\mathrm{NaOCl} 2.5 \%$ and EDTA 17\% or standard protocol (SP), (2) $\mathrm{SP}+\mathrm{Nd}$ :YAG lasing (dried RC), (3) NaOCl 2.5\% + Nd:YAG lasing (dried RC), (4) SP + Nd:YAG lasing (wet RC), or (5) $\mathrm{NaOCl} 2.5 \%+\mathrm{Nd}$ :YAG lasing (wet RC). Groups $1 \mathrm{r}$ to $5 \mathrm{r}$ consisted of the same filled teeth with resected apices up to the most apical point of the preparation length. Resection was performed after the first CFP measurement. Roots were filled with cold lateral condensation. CFP was used to assess minimum, mean flow and maximum pore diameters after $48 \mathrm{~h}$, and immediately after these measurements, including root resection. Statistics were performed by using nonparametric tests $(p>0.05)$. An additional three roots per group were submitted to SEM of the RC wall. Results: Through-and-through leakage was observed in all groups. Statistically significant differences were observed in maximum pore diameter: $1 r>3 r$, and $1 r>5 r$; in mean flow pore diameter: $1 \mathrm{r}>2 \mathrm{r}, 2 \mathrm{r}<4 \mathrm{r}(p<0.05)$. Typical Nd:YAG glazing effects were observed when the smear layer was present and exposed to the laser fiber (i.e., in the groups without use of EDTA) or when the fiber tip made direct contact with a smear-layer free RC wall. Conclusions: The reduction in through-and-through leakage is significantly higher with the Nd:YAG laser as smear-layer modifier than when smear layer is removed with an EDTA rinsing solution.
\end{abstract}

\section{Introduction}

$\mathrm{T}$ HE ND:YAG LASER has now been evaluated for its application in endodontics over a number of years. An abundant number of studies have shown that direct $\mathrm{Nd}$ :YAG laser irradiation on dentin surfaces produces melting and recrystallization, which, in turn, causes a reduction in dentin permeability. ${ }^{1-7}$ In view of "dentin melting" ${ }^{\text {"8 }}$ and "reduction of the permeability of dentin", 3 a number of investigators have attempted to improve the quality of the apical seal and thus to reduce apical leakage after apical filling. ${ }^{9-15}$ It is clear from these studies that morphologic changes after Nd:YAG laser irradiation can influence the marginal sealing of the root-canal obturation. Data evaluating coronal leakage of root fillings after laser irradiation of the root-canal walls remain scarce. ${ }^{11,16}$
To prevent the ingress of new pathogens from the oral cavity and to maintain the success of the endodontic procedure, an adequate three-dimensional obturation and coronal seal, after meticulous cleaning and shaping of the root-canal space, are required. ${ }^{17,18}$ The removal of the smear layer plays an important role in this success, with the use of EDTA as a determining parameter. EDTA rinsing solution used as a final rinse provides effective smear-layer removal. ${ }^{19,20}$ Irrigants that can remove the smear layer allow penetration of sealers into dentinal tubules. ${ }^{21,22}$ Sealer penetration may serve an important role by preventing reinfection and by incarcerating and depriving residual microorganisms of a nutrient source. $^{23,24}$ It will increase the interface between materials and dentin, thus improving the sealing ability and retention of the material by mechanical interlocking. ${ }^{25}$

Department of Operative Dentistry and Endodontology, Ghent Dental Laser Centre, Dental School, Ghent University, Ghent, Belgium. 
The obturation technique apparently plays a minor role in the degree of interlocking and penetration of the sealer into the dentinal tubules. ${ }^{25}$ A first aim of this study was hence to investigate whether smear-layer removal by the use of EDTA in association with the Nd:YAG laser would affect microleakage differently. With limited information available regarding the morphologic changes after Nd:YAG laser irradiation in root canals after irrigation with both $\mathrm{NaOCl}$ and EDTA, SEM analysis was performed in addition on all experimental groups to visualize the effect of the cleaning protocol on the root-canal walls.

Evidence suggests that laser-activated irrigation with an erbium laser may induce cavitation when the laser is activated in the irrigant itself. ${ }^{26,27}$ The result is an enhanced removal of the smear layer as compared with passive irrigation with the endodontic needle. ${ }^{28}$ Laser interaction with matter generating cavitation and subsequent pressure waves has also been described for the Nd:YAG. ${ }^{29}$ A second aim of this study was then to investigate the effect of laser activation into the root-canal irrigant on root-canal wall cleanliness and the final seal obtained with the root filling.

To eliminate the effect of morphologic changes at the most apical preparation point just above the apical constriction on the seal of the root filling, ${ }^{16}$ all roots were also tested on through-and-through leakage after resection of the root tip, thus exposing the gutta-percha root filling.

\section{Materials and Methods}

\section{Tooth selection}

This study used 75 extracted human straight single-rooted teeth with mature apices for the leakage experiments (five groups of 15 teeth) and 15 teeth (five groups of three teeth) for scanning electron microscopic evaluation. The experimental protocol was approved by the ethical committee of the Ghent University Hospital (Belgium) (2008/186). All teeth were stored in $10 \%$ formalin until each experimental subgroup was complete. The preservation time before root-canal treatment did not exceed 6 weeks. To exclude teeth with multiple root canals, all teeth were radiographed from two angles before root-canal treatment. Organic debris was removed by submerging the teeth in $2.5 \%$ sodium hypochlorite for $8 \mathrm{~h}$. Subsequently, they were washed with tap water for $1 \mathrm{~h}$ and stored in saline solution until used.

\section{Root-canal treatment}

Crowns were removed $2 \mathrm{~mm}$ above the cementoenamel junction by using a high-speed fissure burr and water spray. After gross removal of pulp tissues, a size 10 Flexofile (Dentsply Maillefer, Baillaigues, Switzerland) was introduced into the canal until it could be seen in the major apical foramen. The working length was determined by subtracting $1 \mathrm{~mm}$ from this length. The root canals were prepared by means of a crowndown/stepback technique, by one operator. The coronal half of the root canals was preflared with Gates Glidden drills (Dentsply Maillefer) in a larger-to-smaller sequence (numbers 4-3-2), and the canals were copiously irrigated with $2.5 \%$ sodium hypochlorite solution by using a 27-gauge endodontic needle (Monoject; Sherwood Medical, St. Louis, MO). The apical half of the canal was then prepared with the stepback technique up to a master file size 40 . The canals were dried with paper points, and the patency of the apical foramen was confirmed with a size 10 Flexofile. The assignment of the teeth to the specific experimental groups was determined by the final cleaning of the root canal: a final rinse with $3 \mathrm{ml}$ 17\% EDTA solution (Pulpdent EDTA Solution $17 \%$; Pulpdent Corporation, Watertown, MA) for $3 \mathrm{~min}$, or lasing with the Nd:YAG laser (FidelisPlus II; Fotona, Ljubljana, Slovenia), or the combination of a $17 \%$ EDTA rinse followed by Nd:YAG lasing. When the EDTA solution was used as final rinse, an additional rinse with $2.5 \% \mathrm{NaOCl}$ was performed.

This protocol resulted in five groups of 15 teeth (Groups 1 to 5): (1) $2.5 \% \mathrm{NaOCl}$ rinses during root-canal preparation and a final rinse with 17\% EDTA (standard protocol), followed by the rinsing out of the 17\% EDTA with $2.5 \%$ $\mathrm{NaOCl}$; (2) standard protocol $+\mathrm{Nd}: \mathrm{YAG}$ lasing in a dried root canal; (3) $2.5 \% \mathrm{NaOCl}$ rinses during root-canal preparation followed by $\mathrm{Nd}$ :YAG lasing in a dried root canal; (4) standard protocol $+\mathrm{Nd}$ :YAG lasing in a wet root canal; and (5) $2.5 \% \mathrm{NaOCl}$ rinses during root-canal preparation followed by Nd:YAG lasing in a wet root canal. These five groups consisted of the nonresected teeth. The same teeth were then horizontally resected up to the most apical point of the canal preparation after leakage measurement, so that the root-canal filling was exposed, and leakage could be performed in the resected roots. This resulted in an additional five experimental groups: Groups $1 \mathrm{r}$ to $5 \mathrm{r}$.

\section{Laser treatment}

Root canals in the lased groups were irradiated with an Nd:YAG laser $(1.064 \mu \mathrm{m})$ (FidelisPlus II; Fotona) with a flexible fiber (diameter, $300 \mu \mathrm{m}$ ) at $1.5 \mathrm{~W}, 15 \mathrm{~Hz}, 100 \mathrm{~mJ}$. The flexible fiber was inserted into the root canal $1 \mathrm{~mm}$ short of the working length. During irradiation, the fiber tip was moved in a spiral motion along the root-canal walls. The procedure was repeated 4 times for $5 \mathrm{~s}$, with a time interval of $20 \mathrm{~s}$. All apical foramina remained patent (control with a file ISO 15).

\section{Root-canal filling}

All root canals were dried with paper points before filling. A standard size gutta-percha cone (Dentsply Maillefer) that matched the master apical file was fitted to the working length with tug back. Root canal sealer AH 26 (Dentsply Detrey, Konstanz, Germany) was mixed according to the manufacturer's instructions and placed in the canal with the guttapercha to working length. The master cone was then coated again with root-canal sealer and gently seated at the working length.

Lateral condensation was carried out by using size 20 and 25 accessory gutta-percha cones with endodontic finger spreaders (Dentsply Maillefer) placed, in the first instance, to within $1 \mathrm{~mm}$ of the working length. The gutta-percha cones coated with sealer were laterally condensed until they could not be introduced $>3 \mathrm{~mm}$ into the root canal. After obturation, the gutta-percha was removed from the coronal cavity up to the level of the cementoenamel junction with a warm instrument (PK Thomas Waxing Instrument, PKT-2; Hu Friedy, Leimen, Germany) and vertically condensed with Machtou pluggers (Dentsply Maillefer). 
After the root-filling procedure, a small cotton pellet was sealed in the access cavity of all root-filled teeth by using Ketac-Fil (3MEspe, Seefeld, Germany). The samples were then stored in Vacutainers at $80 \%$ relative humidity for $48 \mathrm{~h}$ at $37^{\circ} \mathrm{C}$ (start of the first capillary-flow porometry measurements). Before storing the teeth, radiographs were taken from the buccal and mesial sides of every tooth.

\section{Measurement of capillary flow}

Capillary-flow porometry (CFP, 1200 A; PMI, New York, NY) provides fully automated through-pores analysis including bubble-point pressure, pore-size distribution, and mean pore size. A wetting liquid (Galwick: 15.9 dynes/cm, PMI) was used to fill the pores of the sample. The fully wetted teeth were attached in the sample chamber (Tubepack; Legris Connectic, France), with adhesive epoxy (Loctite 3430; Loctite, Kontich, Belgium), after which the sample chamber was sealed. Gas was then allowed to flow into the chamber behind the sample. When the pressure reaches a point that can overcome the capillary action of the fluid within a pore (maximum pore), the equivalent bubble-point pressure has been found. After determination of the bubble-point pressure, the pressure is increased, and the flow is measured until all pores are empty, and the sample is considered dry. Pressure ranges from 0 to 200 PSI, and the pore size range that can be measured lies between 0.035 and $500 \mu \mathrm{m}$. Measurements for this study were performed at VITO (Vlaamse Instelling voor Technologisch Onderzoek, Mol, Belgium).

After $48 \mathrm{~h}$, all nonresected teeth were measured after removal of the Ketac-Fil filling and cotton pellet, to assess the minimal, mean, and maximal through-pore diameters of each experimental tooth. Voids responsible for leakage were supposed to be present between the root-canal filling and the root itself.

A second series of measurements was performed after resection of the root end up to the most apical point of the preparation length, so that the gutta-percha was exposed. In most cases, this resulted in the resection of at least $1 \mathrm{~mm}$ from the physiological apex and almost $2 \mathrm{~mm}$ from the root tip seen as the radiologic apex on a radiograph. The resection was performed with a diamond-wheel saw. After this pro- cedure, all teeth (now Groups 1r to $5 r$ ) were subjected again to CFP, so a comparison could be made from teeth with an apical constriction and those in which the apical gutta-percha is exposed, as is the case with resected teeth.

\section{Statistical analysis}

Results from both methods were analyzed statistically by using nonparametric tests: comparison between the leakage results according to the different additional cleaning protocols were made with Kruskal-Wallis and Mann-Whitney $U$ tests. The level of significance was set at 0.05 .

\section{Scanning electron microscopic evaluation}

Three additional teeth from each experimental group were analyzed with SEM. ${ }^{30}$ These teeth were split longitudinally in the buccolingual plane. The samples were then dehydrated in ascending series of aqueous ethanol, critically point dried with liquid $\mathrm{CO}_{2}$, sputter coated with gold (JEOL JFC1200; JEOL, Japan), and examined under the scanning electron microscope (JEOL JSM-5600-LV; JEOL, Japan).

Representative microphotographs were taken at various magnifications of the apical, middle, and coronal third of the roots. SEM pictures at $\times 2,000$ magnification were evaluated by an independent blinded investigator.

\section{Results}

\section{Leakage assessment by means of capillary-flow porometry}

Measurements were obtained for each sample at each point in time, confirming the presence of through pores regardless of which root-canal wall cleaning protocol was being tested. Exact values for minimum, mean flow, and maximum pore diameters (range and median) of each sample were obtained. The results of the study are summarized in Table 1.

\section{Leakage assessment: no laser treatment versus laser treatment}

No statistically significant differences were found between Groups 1 through 5 of the nonresected teeth. In the group of

Table 1. Range and Median of Minimum, Mean Flow, and Maximum Pore Diameters by Root End Filling Material at 48 H (Groups 1 to 5) ANd Then ImMediately after Root Resection (Groups 1R to 5R)

\begin{tabular}{|c|c|c|c|c|c|c|}
\hline \multirow[b]{2}{*}{ Group } & \multicolumn{2}{|c|}{ Minimum pore diameter $(\mu m)$} & \multicolumn{2}{|c|}{ Mean flow pore diameter $(\mu m)$} & \multicolumn{2}{|c|}{ Maximum pore diameter $(\mu m)$} \\
\hline & Range & Median & Range & Median & Range & Median \\
\hline 1 & $0.069-0.183$ & 0.0870 & $0.078-0.277$ & 0.1080 & $0.152-0.483$ & 0.3020 \\
\hline 2 & $0.069-0.161$ & 0.0890 & $0.082-0.380$ & 0.1390 & $0.094-0.438$ & 0.3090 \\
\hline 3 & $0.069-0.236$ & 0.1170 & $0.083-0.333$ & 0.1570 & $0.095-0.474$ & 0.2500 \\
\hline 4 & $0.069-0.234$ & 0.0980 & $0.086-0.321$ & 0.1500 & $0.143-0.718$ & 0.2490 \\
\hline 5 & $0.069-0.236$ & 0.0750 & $0.074-0.331$ & 0.1370 & $0.088-0.422$ & 0.2090 \\
\hline $1 \mathrm{r}$ & $0.069-0.209$ & 0.0840 & $0.090-0.332$ & 0.1180 & $0.162-0.976$ & 0.3080 \\
\hline $2 \mathrm{r}$ & $0.069-0.178$ & 0.0840 & $0.078-0.254$ & 0.1020 & $0.134-0.466$ & 0.3060 \\
\hline $3 r$ & $0.069-0.159$ & 0.0850 & $0.077-0.240$ & 0.1350 & $0.138-0.448$ & 0.2130 \\
\hline $4 \mathrm{r}$ & $0.068-0.305$ & 0.1020 & $0.073-0.424$ & 0.2100 & $0.234-0.463$ & 0.3020 \\
\hline $5 \mathrm{r}$ & $0.069-0.248$ & 0.0730 & $0.077-0.464$ & 0.1480 & $0.186-0.850$ & 0.2830 \\
\hline
\end{tabular}

Groups 1 to 5 consist of nonresected roots. Control group: 1. irrigation with NaOCl 2.5\% and EDTA 17\% or standard protocol (SP); Dry lasing protocol: 2. SP + Nd:YAG lasing (dried root canal, RC), 3. NaOCl $2.5 \%+\mathrm{Nd}$ :YAG lasing (dried RC); wet lasing protocol: 4 . $\mathrm{SP}+\mathrm{Nd}: \mathrm{YAG}$ lasing in EDTA (wet RC), 5 . $\mathrm{NaOCl} 2.5 \%+\mathrm{Nd}: \mathrm{YAG}$ lasing in $\mathrm{NaOCl}$ (wet RC). 
the resected roots, statistically significant differences in mean flow pore diameter were observed between Groups $1 \mathrm{r}$ and $2 \mathrm{r}$ ( $p=0.026)$, with Group 1r having the largest mean flow pore diameter. Statistically significant differences in maximum pore diameter were observed between Groups $1 \mathrm{r}$ and $3 \mathrm{r}$ $(p=0.016)$, and $1 \mathrm{r}$ and $5 \mathrm{r}(p=0.023)$, with Group 1r having the largest maximum pore diameter in both comparisons. No significant differences were seen between Groups $1 \mathrm{r}$ and $4 \mathrm{r}$.

Groups $1 r$ to $5 r$ consist of the filled teeth of Groups 1 to 5 with resected apices up to the most apical point of the preparation length and exposing the root-canal filling.

\section{Leakage assessment: dry protocol versus wet lasing protocol}

No statistically significant differences were found between any groups of the nonresected teeth. Statistically significant differences in mean flow pore diameter were observed between Groups $2 \mathrm{r}$ and $4 \mathrm{r}(p<0.003)$, with Group $4 \mathrm{r}$ having the largest mean flow pore diameter. No significant differences were seen between Groups 3r and 5r.

\section{Leakage assessment: nonresected roots and resected roots}

No statistically significant differences were found between the groups with and without root tip and with identical cleaning protocols.

\section{Scanning electron microscopic evaluation}

Representative images of the smear layer after conventional preparation supported with $\mathrm{NaOCl}$ irrigation and followed by a final rinse of EDTA are shown in Fig. 1 (Groups 1 and 1r). Abundant smear-layer removal is seen in the coronal (Fig 1a) and middle third (Fig. 1b). In the apical part, only a limited part of the dentinal tubules is opened (Fig. 1c and d), and an abundant smear layer is still present. Figure 2 shows the effect of exposure of the root-canal wall
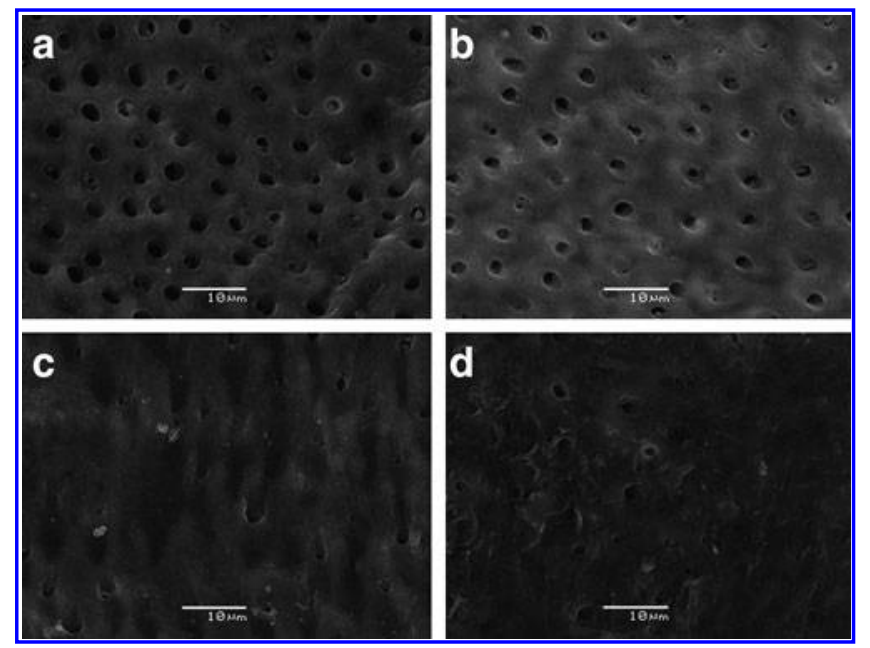

FIG. 1. SEM photos of root-canal walls cleaned with $2.5 \%$ $\mathrm{NaOCl}$ during root canal preparation and a final rinse of $17 \%$ EDTA. Smear layer is removed in the coronal (a) and middle third (b) of the root canal. In the apical third, smear layer is still present (c, d). after final rinsing with EDTA to the Nd:YAG laser (Groups 2 and 2r). Areas with open tubules could be detected, especially in the coronal and middle third (Fig. 2a) and less in the apical third. Local areas of melted and evaporated dentin were found in areas where the fiber had touched the root canal walls (Fig. $2 b$ and d). Localized areas with removal of root-canal wall dentin without glazing or melting effect were observed in the middle third (Fig. 2c). Figure 3a shows the classic image of the morphologic effect of a smear layer exposed to the Nd:YAG laser (image taken in the apical third); areas of melting, glazing, and recrystallization were seen in all thirds (Groups 3 and 3r). When the Nd:YAG laser was activated in the EDTA solution, clean root-canal walls to the same extent as when the canal was rinsed with EDTA solution were found, although the root-canal walls showed irregular dentin surfaces with round cuffs around the orifices of the dentinal tubules (Fig. 4a-c) (Groups 4 and 4r). These rounded areas, still covered with a smear layer, were also observed (Fig. 4d). In the group in which the Nd:YAG laser was used in $\mathrm{NaOCl}$ (Groups 5 and 5r), a smear layer was present at all sites, except for some areas where the fiber touched the root-canal wall, and a direct interaction with the root-canal wall led to localized removal of dentin and signs of carbonization and melting (Fig. 3b-d).

\section{Discussion}

In the present study, microleakage was investigated in association with different lasing and cleaning protocols. A comparison was also made for identical cleaning protocols between nonresected roots and resected roots to avoid the impact of root-canal wall modification with the Nd:YAG laser at the level of the apical stop and the apical constriction. In previous studies, it was demonstrated that morphologic changes at this point influenced the apical seal in a positive
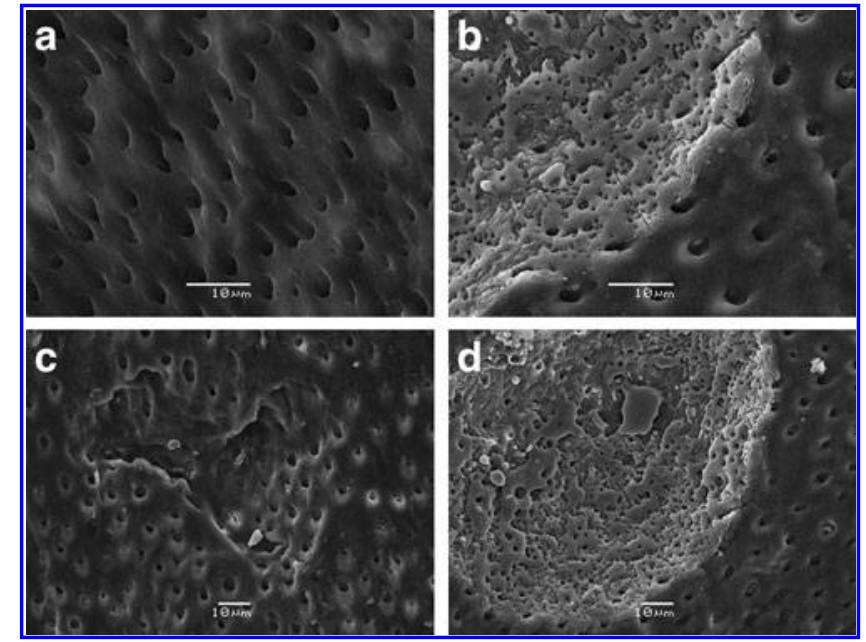

FIG. 2. SEM photos of root-canal walls cleaned with $\mathrm{Nd}$ :YAG after the sole use of $\mathrm{NaOCl}$ as irrigant. When $\mathrm{Nd}$ :YAG is used in dried canals, typical morphologic changes such as glazing, melting, and recrystallization are observed (a). When the fiber is used in wet canals (in $\mathrm{NaOCl}$ ), the effect of the laser is diminished or disappears (c, d). The classic morphologic changes after Nd:YAG laser-root-canal wall dentin interaction are seen when the laser fiber touches the canal wall itself (b). 

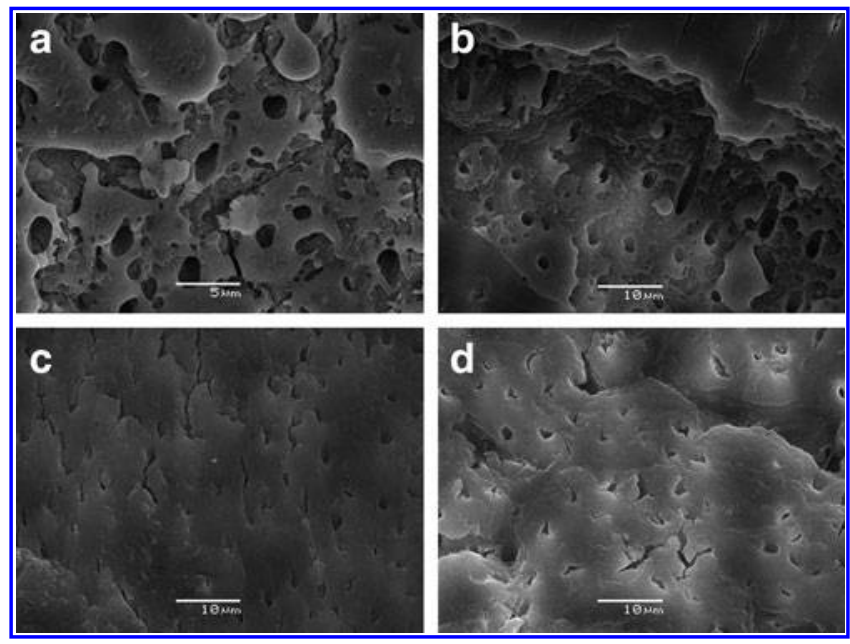

FIG. 3. SEM photos of root-canal walls cleaned with $2.5 \%$ $\mathrm{NaOCl}$ during root-canal preparation and a final rinse of $17 \%$ EDTA, followed by Nd:YAG lasing. Because of the absence of smear layer, the typical morphologic changes associated with Nd:YAG lasing are not seen [(a) photomicrograph in the coronal third]. It is only when the fiber touches the root-canal wall that effects of laser-tissue interaction are observed $(\mathbf{b}-\mathbf{d})$.

way. ${ }^{13,16}$ So the impact of morphologic changes of the rootcanal wall due to the Nd:YAG on the lateral seal of a root filling could be investigated.

Capillary-flow porometry (CFP) was used to assess leakage. The technique has been used extensively in industries worldwide, ranging from membrane filtration to nonwovens to battery industries for R \& D and quality control. The method has been approved by the American Society of Testing and Materials (ASTM Designation 1999). The validity of this technique in dentistry has been verified by De Bruyne

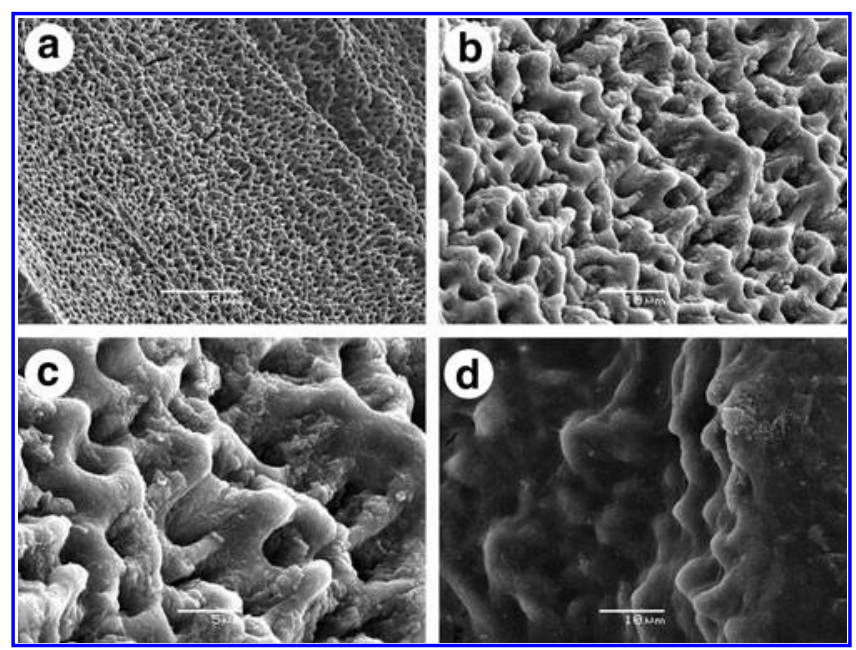

FIG. 4. SEM photos of root-canal walls where the Nd:YAG laser is activated in $17 \%$ EDTA solution. Areas with removal of smear layer $(\mathbf{a}-\mathbf{c})$ and others without are seen $(\mathbf{d})$. The morphology of the root-canal wall is clearly different from the morphologic changes seen in association with $\mathrm{Nd}: \mathrm{YAG}$ lasing: a surface with open protruded dentinal tubules and rounded borders is seen. et al., ${ }^{31,32}$ who consequently introduced this method into endodontics. CFP was chosen as the evaluation method because of its nondestructive nature and the highly reproducible and accurate data it generates. ${ }^{33-35}$ As such, the method can overcome the problem of limited reproducibility and comparability of conventional methods for evaluating leakage. $^{36,37}$

The removal of the smear layer is considered important: its removal results in a proper action of irrigants and intracanal medication, a deeper penetration of root-canal sealer, and more obturated lateral canals. ${ }^{38-41}$ It has been demonstrated that the alternate use of $\mathrm{NaOCl}$ and EDTA irrigants results in smear-layer removal and a dentin surface with open tubules. $^{42}$ This information has to be interpreted with care, as the removal of smear layer in the apical region remains unpredictable. ${ }^{20,43}$ The latter was also the case in the present study (Fig. 1c and d).

Morphologic changes due to Nd:YAG laser irradiation, such as fusion and recrystallization of the dentin, were already demonstrated. ${ }^{1-7,13}$ These changes are confirmed on the SEM images in this study in Group 3 with Nd:YAG lasing, after the $\mathrm{NaOCl}$ supported root-canal preparation (Fig. 3a). For all other groups in which the Nd:YAG was used, the typical signs of glazing, melting, and recrystallization were not observed in the same manner. The effect of the Nd:YAG was seen only in the group with a final EDTA rinse, when a direct contact occurred with the root-canal wall (Fig. 2). Apparently the absence of the smear layer results only in a laser-root-canal wall interaction when the root-canal wall is touched. This means that widespread crystallization, glazing, or melting effects are seen only in situations with abundant smear layer before lasing. Hence, when the smear layer is absent, no evaporation or contraction of the smear layer occurs because of Nd:YAG lasing. It is only when direct contact with the root canal wall with the fiber occurs that the typical morphologic effects are found into the dentin. These alterations in the groups that were dried before laser application are more evident, which confirms the findings of Faria et al. ${ }^{44}$ When the Nd:YAG is used in EDTA solution (Fig. 4), changes are found in the rootcanal walls, although these are of another nature than those after lasing in dried root canals after rinsing with EDTA. Further research is needed to investigate the composition of this altered dentin. $\mathrm{Nd}$ :YAG lasing in $\mathrm{NaOCl}$ has no effect on the smear layer, unless the fiber touches the root-canal wall itself. With the present findings, no evidence supports that laser interaction with matter generates cavitation and subsequent pressure waves when the $\mathrm{Nd}$ :YAG laser is used in the classic endodontic irrigants ( $\mathrm{NaOCl}$ or EDTA). In this study, the limitation of the unilateral emission of the laser beam is also substantiated. Only these areas that are exposed to the laser beam demonstrate the typical Nd:YAG morphologic changes of the root-canal wall. It is only when an abundant smear layer exists and in a dried root canal that widespread glazing is found. Hence, the latter can be the result only of heating of the smear layer, leading to evaporation and contraction. When the energy is high, glazing and melting will occur, with carbonization when the exposure to the Nd:YAG laser beam is too intense or too high for the target tissue.

In the present study, the fiber tip was held $1 \mathrm{~mm}$ short of the working length at the beginning of the lasing procedure 
to respect the clinical situation. He et al., ${ }^{45}$ investigating the temperature at the outer surface of a root after Nd:YAG lasing at different power settings, clearly demonstrated and confirmed what others ${ }^{13,16,44}$ had already pointed out: thermal and morphologic changes seen at the apical third of the roots were greater than those in the coronal third, suggesting that caution should be used when irradiating the apical part of the root, to prevent periodontal injury. They demonstrated that root dentin thickness, especially in the apical third, was an important parameter in the conductance of energy and hence temperature toward the periodontal ligament. ${ }^{45}$ Based on their findings, the insertion of the laser fiber $1 \mathrm{~mm}$ short of the working length, as done in the present setup, does not interfere with the requested laser-tissue interaction and is a safe way to avoid periodontal damage. ${ }^{13,16}$

All samples demonstrated leakage (Table 1). Differences between groups with different cleaning protocols became more obvious when the root tips were resected. In a previous study, a looser seal was observed after Nd:YAG lasing when the smear layer was thoroughly removed. ${ }^{12,16}$ The uniform roughened surface left on the dentin walls after lasing of the smear layer appears to favor cement adhesion, and therefore improves marginal sealing. The morphologic changes, such as melting and the formation of glass droplets, also help in occluding the physiologic apex and limits apical ingress or apical leakage. Only when the root tip is removed is the evaluation of true through-and-through leakage along the root canal walls reliable. In this study, the best seal (mean flow and maximum pore diameters) in the resected groups was provided in Group 3r, when a canal was cleaned with $\mathrm{NaOCl}$ followed by Nd:YAG lasing. Based on the SEM photographs, a better cleaning was found for Groups 2 and 2r. Apparently the effects of smear-layer modification and evaporation with the Nd:YAG ensure a better seal than smear layer-free root-canal walls with open tubules, as a result of the chelating action of EDTA.

An adequate root-canal obturation is important to ensure a long-lasting successful endodontic result. ${ }^{17}$ In a recent study, Hammad et al. ${ }^{46}$ investigated the percentage of volume of voids and gaps in root canals obturated with different obturation materials by using a micro-computed tomography (micro-CT). All teeth were filled with a cold lateral condensation technique, and they found $1.02 \%$ gaps or voids in laterally condensed gutta-percha obturations. The maximum pore diameter is the most important determinant for the quality of the apical seal. The average length of bacteria varies between 0.2 and $1.5 \mu \mathrm{m}$, and toxins are even smaller. ${ }^{47}$ Table 1 shows that none of the fillings in this study will inhibit bacterial passage along the root filling. Important is thus that Nd:YAG lasing not only can help to ensure a better seal because of its interaction with the root canal wall, ${ }^{16,48}$ but also can enable a more profound disinfection of the root canal than with $\mathrm{NaOCl}$ rinses. ${ }^{49} \mathrm{AH} \mathrm{26}$, a hydrophobic resin sealer, was used for the root-canal obturation. de Moura-Netto et al. ${ }^{15}$ demonstrated that the use of a hydrophobic sealer is to be preferred above a hydrophilic resin sealer because of the dehydration and morphologic changes caused by the Nd:YAG laser irradiation. We must emphasize that not every type of sealer is to be used in association with this irradiation. More research, however, is needed in this field.
At present, an ongoing debate exists over how $\mathrm{NaOCl}$ and EDTA may interfere: a number of studies have shown that the combined use of irrigating solutions decreases the effectiveness of chelating agents and might enhance destruction of the dentinal surface. ${ }^{50}$ Furthermore, it might have negative effects on the bond strength of adhesive cement to root-canal dentin. ${ }^{51,52}$ Based on the present findings, an additional advantage of the Nd:YAG laser technique is therefore its cleaning efficacy and low tendency to produce mineral loss from the root-canal dentin. ${ }^{53}$

\section{Conclusion}

The present study confirms the findings of a previous study, ${ }^{16}$ indicating that an added value is to be gained in obtaining a tighter root-canal seal for the Nd:YAG laser as modifier of the root-canal wall in roots where the smear layer has not been removed by EDTA.

Better sealing results are also obtained after the use of the Nd:YAG laser in dried root canals. In this respect, no benefit accrues from using the Nd:YAG laser in irrigation solutions such as $\mathrm{NaOCl}$ and EDTA at the settings of $1.5 \mathrm{~W}, 100 \mathrm{~mJ}$, and $15 \mathrm{~Hz}$, and lasing 4 times for $5 \mathrm{~s}$, with a dwell time of $20 \mathrm{~s}$.

\section{Author Disclosure Statement}

No competing financial interests exist.

\section{References}

1. Dederich, D.N., Zakariasen, K.L., and Tulip, J. (1984). Scanning electron microscopic analysis of canal wall dentin following neodymium-yttrium-aluminium-garnet laser irradiation. J. Endod. 10, 428-431.

2. Levy, G. (1992). Cleaning and shaping the root canal with a Nd:YAG laser beam: a comparative study. J. Endod. 18, 123-127.

3. Stabholz, A., Khayat, A., Weeks, D.A., Neev, J., and Torabinejad, M. (1992). Scanning electron microscopic study of the apical dentine surfaces lased with Nd:YAG laser following apicectomy and retrofill. Int. Endod. J. 26, 288291.

4. Miserendino, L.J., Levy, G.C., and Riziou, I.M. (1995). Effects of Nd:YAG laser on the permeability of root canal wall dentin. J. Endod. 21, 83-87.

5. Anic, I., Tachibana, H., Matsumoto, K. and Qi, P. (1996). Permeability, morphologic and temperature changes of canal dentine walls induced by Nd:YAG, $\mathrm{CO}_{2}$ and argon lasers. Int. Endod. J. 1, 13-22.

6. Altamura, C., Majori, M., Bedini, R., and Filippini, P. (2003). Evaluation of Nd:YAG laser effects on root canal walls. J. Oral Laser Appl. 2, 67-72.

7. de Moura-Netto, C., de Moura, A.A., Davidowicz, H., Aun, C.E., and Antonio, M.P. (2008). Morphologic changes and removal of debris on apical dentin surfaces after Nd:YAG laser and diode laser irradiation. Photomed. Laser Surg. 26, 263-266.

8. Takeda, F.H., Harashima, T., Kimura, Y., and Matsumoto, K. (1998). Comparative study about the removal of smear layer by three types of laser devices. J. Clin. Laser Med. Surg. 16, 117-122.

9. Kimura, Y., Yamazaki, R., Goya, C., Tomita, Y., Yokoyama, K., and Matsumoto, K. (1999). A comparative study on the effects of three types of laser irradiation at the apical stop 
and apical leakage after obturation. J. Clin. Laser Med. Surg. 17, 261-266.

10. Goya, C., Yamazaki, R., Tomita, Y., Kimura, Y., and Matsumoto, K. (2000). Effects of pulsed Nd:YAG laser irradiation on smear layer at the apical stop and apical leakage after obturation. Int. Endod. J. 33, 266-271.

11. Park, D.S., Lee, H.J., and Yoo, H.M. (2001). Effect of Nd-YAG laser irradiation on the apical leakage of obturated root canals: an electrochemical study. Int. Endod. J. 34, 318-321.

12. Carvalho, C.A.T., Valera, M.C., Gown-Soars, S., and De Paulo Eduardo, C. (2002). Effects of Nd:YAG and Er:YAG lasers on the sealing of root canal fillings. J. Clin. Laser Med. Surg. 20, 215-219.

13. Depraet, F.J.H.W., De Bruyne, M.A.A., and De Moor, R.J.G. (2005). The sealing ability of an epoxy resin root canal sealer after Nd:YAG laser irradiation of the root canal. Int. Endod. J. 38, 302-309.

14. Sousa-Neto, M.D., Silva Coelho, F.I., Marchesan, M.A., Alfredo, E., and Silva-Sousa,Y.T. (2005). Ex vivo study of the adhesion of an epoxy-based sealer to human dentine submitted to irradiation with Er:YAG and Nd:YAG lasers. Int. Endod. J 38, 866-870.

15. de Moura-Netto, C., Carvalho, C.F., Moura, A.A.M., Davidowicz, H., and Antoniazzi, J.H. (2007). Influence of $\mathrm{Nd}$ :YAG and diode laser irradiation on apical sealing when associated with $\mathrm{AH}$ Plus and endoRez endodontic cements. Photomed. Laser Surg. 25, 413-417.

16. Meire, M., Mavridou, A., Dewilde, N., Hommez, G., and De Moor, R.J.G. (2009). Longitudinal study on the influence of Nd:YAG laser irradiation on microleakage associated with two filling techniques. Photomed. Laser Surg. 27, 611-616.

17. Hommez, G.M.G, Coppens, C., and De Moor, R.J.G. (2002). Periapical health related to the quality of coronal restorations and root fillings. Int. Endod. J. 35, 680-689.

18. Schilder, H. (2006). Filling root canals in three dimensions. J. Endod. 32, 281-290.

19. Torbeyns, D.R.H., De Bruyne, M.A.A., and De Moor, R.J.G. (2007). A SEM study of smear layer removal in association with $\mathrm{NaOCl}$ and EDTA formulations. Int. Endod. J. 40, 991 $\mathrm{R} 2.38$.

20. Niu, W., Yoshioka, T, Kobayashi, C., and Suda, H. (2002). A scanning electron microscopic study of dentinal erosion by final irrigation with EDTA and $\mathrm{NaOCl}$ solutions. Int. Endod. J. 35, 934-939

21. Kouvas, V., Liolios, E., Vassiliadis, L., Parissis-Messimeris, S., and Boutsioukis, A. (1998). Influence of smear layer on depth of penetration of three endodontic sealers: an SEM study. Endod. Dent. Traumatol. 14, 191-195.

22. Kokkas, A.B., Boutsioukis, A.Ch., Vassiliadis, L.P., and Stavrianos, C.K. (2004). The influence of the smear layer on dentinal tubule penetration depth by three different root canal sealers: an in vitro study. J. Endod. 30, 100-102.

23. Assouline, L.S., Fuss, Z., Mazor, Y., and Weiss, E.I. (2001). Bacterial penetration and proliferation in root canal dentinal tubules after applying dentin adhesives in vitro. J. Endod. 27, 398-400.

24. Weis, M.V., Parashos, P., and Messer, H.H. (2004). Effect of obturation technique on sealer cement thickness and dentinal tubule penetration. Int. Endod. J. 37, 653-663.

25. Mamootil, K. and Messer, H.H. (2007) Penetration of dentinal tubules by endodontic sealer cements in extracted teeth and in vivo. Int. Endod. J. 40, 873-881.
26. Blanken, J.W., and Verdaasdonk, R.M. (2007). Cavitation as a working mechanism of the Er,Cr:YSGG laser in endodontics: a visualization study. J. Oral Laser Appl. 7, 97-106.

27. Blanken, J., De Moor, R.J.G., Meire, M., and Verdaasdonk, R. (2009). Laser induced explosive vapor and cavitation resulting in effective irrigation of the root canal. Part 1: A visualization study. Lasers Surg. Med. July 28. 41, 514-519.

28. De Moor, R.J.G., Blanken J., Meire, M., and Verdaasdonk, R. (2009) Laser induced explosive vapor and cavitation resulting in effective irrigation of the root canal. Part 2: evaluation of the efficacy. Lasers Surg. Med. 41, 520-523.

29. Levy, G., Riziou, I., Friedman, S., and Lam, H. (1996). Pressure waves in root canals induced by Nd: YAG laser. J. Endod. 22, 81-84.

30. Delmé, K.I.M., and De Moor, R.J.G. (2007). Scanning electron microscopic evaluation of enamel and dentin surfaces after Er: YAG laser preparation and laser conditioning. Photomed. Laser Surg. 25, 393-401.

31. De Bruyne, M.A.A., De Bruyne, R.J.E., Rosiers, L., and De Moor, R.J.G. (2005). Longitudinal study on microleakage of three root-end filling materials by fluid transport method and by capillary flow porometry. Int. Endod. J. 38, 129-136.

32. De Bruyne, M.A., De Bruyne, R.J., and De Moor, R.J.G. (2006). Capillary flow porometry to assess the seal provided by root-end filling materials in a standardized ad reproducible way. J. Endod. 32, 206-209.

33. Jena, A., and Gupta, K. (2002). Characterization of pore structure of filter media. Fluid Particle Separation J. 14, 227-241.

34. Gupta, V., and Jena, A.K. (1999). Substitution of alcohol in porometers for bubble point determination. Adv. Filtrat. Separat. Technol. 13b, 833-844.

35. Mayer, E. (2002) Porometry characterization of filter media. Filtration News 20, 1-7.

36. Wu, M-K., and Wesselink, P.R. (1993). Endodontic leakage studies reconsidered. Part I: methodology, application and relevance. Int. Endod. J. 26, 37-43.

37. Pommel, L., Jacquot, B., and Camps, J. (2001). Lack of correlation among three methods for evaluation of apical leakage. J. Endod. 27, 347-350.

38. Berutti, E., Marini, R., and Angeretti, A. (1997). Penetration ability of different irrigants into dentinal tubules. J. Endod. 23, 725-727.

39. Eldeniz, A.U., Erdemir, A., and Belli, S. (2005). Shear bond strength of three resin based sealers to dentin with and without the smear layer. J. Endod. 31, 293-296.

40. Saleh, I.M., Ruyter, I.E., Haapasalo, M., and Orstavik, D. (2002). The effects of dentine pretreatment on the adhesion of root-canal sealers. Int. Endod. J. 35, 859-866.

41. Villegas, J.C., Yoshioka, T., Kobayashi, C., and Suda, H. (2002) Obturation of accessory canals after four different final irrigation regimes. J. Endod. 28, 534-536.

42. O'Connell, M.S., Morgan, I.A., Beeler, W.J., et al. (2000). A comparative study of smear layer removal using different salts of EDTA. J. Endod. 26, 739-743.

43. Kuah, H-G., Lui, J-N., Tseng, P.S.K., and Chen, N-N. (2009) The effect of EDTA with and without ultrasonics on removal of the smear layer. J. Endod. 35, 393-396.

44. Faria, M.I., Souza-Gabriel, A.E., Marchesan, M.A., SousaNeto, M.D., and Silva-Sousa, Y.T. (2008). Ultrastructural evaluation of radicular dentin after Nd:YAG laser irradiation combined with different chemical substances. Gen. Dent. 56, 641-646. 
45. He, H., Yu, J., Song, Y., Lu, S., Liu, H., and Liu, L. (2009). Thermal and morphological effects of the pulsed Nd:YAG laser on root canal surfaces. Photomed. Laser Surg. 27, 235-240.

46. Hammad M., Qualthrough, A., and Silikas, N. (2009). Evaluation of root canal obturation: a three-dimensional in vitro study. J. Endod. 35, 541-544.

47. Hobot, J.A. (2002). Bacterial Ultrastructure, in: Molecular Microbiology. J.A. Hobot (ed.). London: Academic Press, p. 7.

48. De Moor, R.J.G, Torbeyns, D., and Meire, M. (2009). Lasers in endodontics: Part 2: root canal wall cleanliness and modification. ENDO 3, 19-33.

49. Meire, M., and De Moor, R.J.G. (2007). Lasers in endodontics: laser disinfection, an added value? ENDO 1, 159-172.

50. Dogan, H., and Qalt, S. (2001). Effects of chelating agents and sodium hypochlorite on mineral content of root dentin. J. Endod. 27, 578-580.

51. Ari, H., and Erdemir, A. (2005). Effects of irrigation solutions on mineral content of root canal dentin using ICP-AES technique. J. Endod. 31, 187-189.
52. Erdemir, A, Ari, H., Güngünes, H., and Belli, S. (2004). Effect of medications for root canal treatment on bonding to root canal dentin. J. Endod. 30, 113-116.

53. Gurbuz, T., Ozdemir, Y., Kara, N., Zehir, C., and Kurudirek, M. (2008). Evaluation of root canal dentin after Nd:YAG laser irradiation and treatment with five different irrigation solutions: a preliminary study. J. Endod. 34, 318321.

Address correspondence to:

Prof. Dr. Roeland De Moor Department of Operative Dentistry and Endodontology Dental School, Ghent Dental Laser Center Ghent University, Ghent University Hospital De Pintelaan 185/P8 B-9000 Gent Belgium

E-mail: roeland.demoor@ugent.be 